\title{
Raman spectroscopy of DNA-wrapped single-walled carbon nanotube films at 295 and $5 \mathrm{~K}$
}

\author{
V.A. Karachevtsev and A.Yu. Glamazda \\ B. Verkin Institute for Low Temperature Physics and Engineering of the National Academy of Sciences of Ukraine \\ 47 Lenin Ave., Kharkov 61103, Ukraine \\ E-mail: karachevtsev@ilt.kharkov.ua
}

Received January 21, 2010

\begin{abstract}
Resonance Raman spectra of DNA-wrapped single-walled carbon nanotubes films were studied at 5 and $295 \mathrm{~K}$ in the range of radial-breathing $\left(175-320 \mathrm{~cm}^{-1}\right)$ and tangential $\left(1520-1625 \mathrm{~cm}^{-1}\right)$ modes. The spectra were compared with those of nanotubes in bundles. At $5 \mathrm{~K}$ in the spectrum of film an upshift of bands regard to their spectrum at high temperature and the intensity redistribution among bands of two samples were observed. The magnitude of this upshift depends on the nanotube type. The influence of temperature lowering, the environment and the electron-phonon coupling on the Raman spectrum of nanotubes are discussed.
\end{abstract}

PACS: 78.67.Ch Nanotubes.

Keywords: single-walled carbon nanotubes, DNA, Raman scattering, electron-phonon coupling, low temperatures.

\section{Introduction}

A single-walled carbon nanotube (SWNT) is a quasione-dimensional crystal characterized with unique physical and chemical properties, which can be used in various fields of nanotechnology $[1,2]$. Despite a rather rigid mechanical frame of nanotubes, the environment affects essentially their physical properties. Therefore, study of this environment influence is actual and important at present in carbon nanotube science.

By volume synthesis, carbon nanotubes are obtained in the form of bundles, which appeared due to the strong internanotube interaction caused mainly by van der Waals forces. Splitting of these bundles into individual nanotubes and the following hindrance of nanotube aggregation was not turned out such a simple task as expected. Efforts applied to solve this problem leaded to nanotechnology based on the ultrasonication of nanotubes in water with a surfactant and the following ultracentrifugation. Stable aqueous suspension of individual nanotubes is prepared with adding of charged or neutral surfactants or water-soluble polymers which are prevent nanotubes from sticking after ultrasonication treatment [3]. Besides the need for isolated SWNTs in aqueous solutions, it is desirable to obtain them in the film which extends of range for practical use, for example, as sensors. In this respect, polymers are more promising. Some of them are able to wrap around the tube and to be adsorbed to the tube surface after deposition on the substrate and drying. Investigations performed in this direction for last ten years have shown that the most effective polymer is DNA as it has hydrophobic and hydrophilic parts in the polymer structure simultaneously and, due to its helical form, the polymer can wrap around the nanotube [4]. This model proposes that hydrophobic nitrogen bases are adsorbed to the nanotube surface via $\pi-\pi$ stacking and the hydrophilic sugar-phosphate backbone is directed to water $[3,4]$. It should keep in mind that DNA is a negatively charged polymer and that charged groups effect on its interaction with the nanotube as well. This process takes place not only during ultrasonication but after this treatment too, due to self-ordering to find its optimal energetic position on the nanotube surface in water. As experiments revealed, the process can proceed for weeks and even months $[5,6]$.

Upon the preparation of nanotube films from water suspension with DNA, a composite is formed in which individual nanotubes or small bundles are separated with the polymer $[7,8]$. This conclusion is confirmed with the observation of the luminescence of SWNT:DNA film, which suggests the presence of individual tubes as in nanotube bundles a luminescence is quenched because of the semiconducting SWNT contact with metallic ones [7].

Raman spectroscopy has been used extensively to characterize carbon nanotubes since it can probe both the phonon spectrum and the electronic structure through the resonant effect. Resonance Raman (RR) spectroscopy is characterized by the strong enhancement in the intensity of the scattered light appeared in spectra. RR spectrum of carbon 
nanotubes is observed when the energy of the incident laser matches the energy separation $E_{i i}$ between the peaks of van Hove singularities in the valence and conduction bands (see, for example, [9-13]). Since $E_{i i}$ depends on the nanotube structure, nanotubes of different chiralities or conductivity will be in resonance with laser energies. The sensitivity of this informative method is sufficiently high and researchers have opportunity to observe the spectrum of the individual nanotube (see, for example, [10]). This comparatively low cost method permits to determine diameters, chirality, metallic or semiconducting types of conductivity and to study the effect of temperature and the environment on nanotube properties.

The intensity of bands in the RR spectrum can weaken (even disappear) or, on the contrary, the intensity of other bands may enhance, depending on the position of the electron energy transition relatively to the laser energy (the resonance condition). Thus, there is a small range of laser energy (resonance window) in which intensity of SWNT band in the RR spectrum can attain a maximal value. It is known that the width and shape of this resonance window depends on the nanotube index chirality $(n, m)$, environment, bundling or individual form, nanotubes are in solution or in film, type of surfactant (see, for example, [12]). It should be added that, due to changes of the sample temperature or environment, a shift of electronic levels occurs and, as a result, these levels leave the resonance window or enter it. Thus, even weak interaction between the nanotube and molecule/polymer can be followed not only with the band shift but with a rather strong transformation of the spectrum intensity. Under study of the temperature influence on RR spectra of carbon nanotubes, a priori, such an important factor as the electron-phonon interaction should be accounted too [14]. It should be added that recent Raman scattering experiments on metallic SWNTs revealed a very strong electron-phonon coupling in these nanotubes while applying an electrochemical gate voltage [15-17] or combining Rayleigh scattering with RR spectroscopy [18]. This coupling is caused by the predicted Kohn anomaly (KA) in their phonon dispersion [19-23].

Earlier, RR spectra of carbon nanotubes in bundles [2427] or of isolated SWNT [28-30] were investigated at low temperatures in order to get information on thermal characteristics of this nanomaterial and to study low temperature effects on nanotube structural and energy parameters. It was shown that the temperature dependence of Raman frequencies of SWNT is mainly determined by the anharmonic terms in the bond potential energy.

This work presents results of the experimental study in which effects of low temperature on RR spectrum in the range of radial-breathing (RBM) $\left(175-320 \mathrm{~cm}^{-1}\right)$ and tangential $\left(1520-1625 \mathrm{~cm}^{-1}\right)$ modes $(G$ mode) of SWNT film in DNA environment were elucidated as well as this nanotube film thermal properties were compared with those of nanotubes in bundles.

\section{Experimental details}

SWNTs produced by HiPCO method were purified by controlled thermal oxidation followed by $\mathrm{HCl}$ treatment [31]. An aqueous suspension of SWNTs was prepared using single-stranded DNA. A single-stranded polymer was obtained from a double-stranded one (extracted from chicken erythrocytes, Reanal, Budapest, Hungary) by melting at $90{ }^{\circ} \mathrm{C}$ and quick cooling to ice temperature. SWNT aqueous suspension with DNA was prepared by $30 \mathrm{~min}$ ultrasonication of nanotubes and centrifugation (1 hour, $120000 \mathrm{~g})$. Our electrophoresis estimation of the DNA fragmentation after sonication gave the mean length of the fragment within 200-300 base pairs. The SWNT:DNA concentration ratio was 1:2. Nanotube films for Raman experiments were obtained by dropping DNA-wrapped SWNT suspension onto the quartz substrate, washing the excess DNA and drying in warm air.

Our previous studies [7] and works of other authors [8] showed that SWNTs covered with DNA aggregate into small bundles as the film begins to dry but DNA precludes the direct nanotube-nanotube aggregation in this film. Thus, the bundles in SWNT:DNA film are different from the usual SWNT bundles, and this is confirmed by observation of luminescence of SWNT:DNA in a film, which suggests the presence of individual tubes (or small bundles).

Raman experiments were performed in the $90^{\circ}$ scattering configuration relatively to the laser beam, using the $632.8 \mathrm{~nm}(1.96 \mathrm{eV})$ excitation from a $\mathrm{He}-\mathrm{Ne}$ laser $(15 \mathrm{~mW})$. The scattered light was analyzed with a double monochromator (reverse dispersion $3.5 \AA / \mathrm{mm}$ ) and detected with a thermocooled CCD camera. In our spectral measurements, the peak position of RBM bands and $G^{+}$ band of SWNTs in films and in bundles was determined with an accuracy no worse than $0.5 \mathrm{~cm}^{-1}$. Such high accuracy was possible since the frequency positions of the plasma lines from the $\mathrm{He}-\mathrm{Ne}$ laser in the vicinity of these bands were used for internal calibration of our spectrometer. Low temperature studies were carried out with using the optical cryostat (ILTPE, Ukraine) in helium vapors at $5 \mathrm{~K}$.

\section{Results and discussion}

\section{Resonance Raman spectra of SWNT bundles and SWNT:DNA films at $295 \mathrm{~K}$}

Figure 1 presents two the most characteristic fragments of RR spectrum of SWNT bundles and SWNT:DNA film obtained upon deposition of individual nanotubes from aqueous suspension $(T=295 \mathrm{~K})$. Two spectral ranges are shown which contain the low frequency region between $175-320 \mathrm{~cm}^{-1}$ (RBM bands) (Fig. 1,a) and the high frequency range (Fig. 1,b) between $1520-1625 \mathrm{~cm}^{-1}$ attributed to the tangential band ( $G$ band) frequency. 

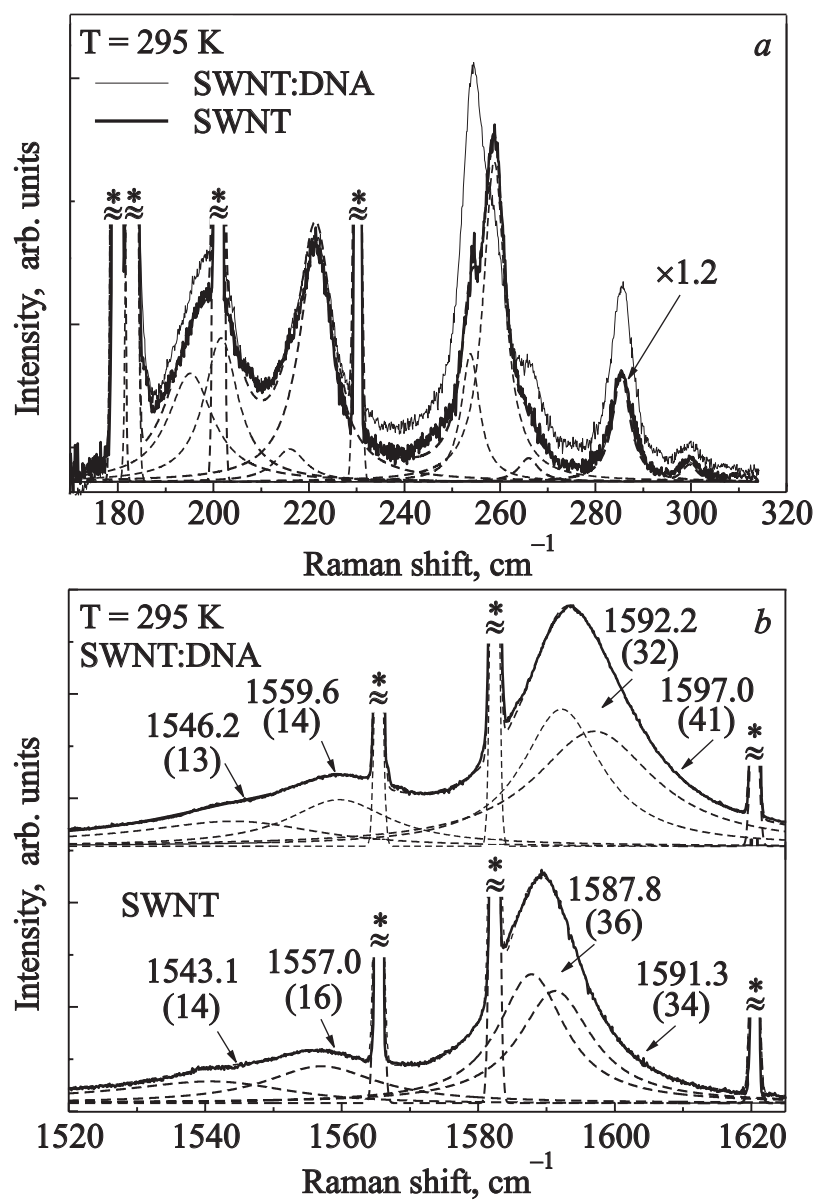

Fig. 1. Raman spectra in range of RBM (a) of SWNT bundles (multiplied by 1.2) (bold line) and SWNT:DNA film (thin line) and $G$ mode $(b)$ at $295 \mathrm{~K}$. Each experimental spectrum obtained at $\lambda_{\text {exc }}=632.8 \mathrm{~nm}$ laser excitation was fitted with curves described with sum of Lorentzians (dashed line) and BWF (a lowfrequency band of $G$ mode) functions. Value of band peak position $\left(\mathrm{cm}^{-1}\right)$ and its area (in brackets) is indicated in this Figure close to the band location. Peaks labeled $\left\langle{ }^{*}\right\rangle$ correspond to the plasma line of the laser.

\section{Radial-breathing mode of nanotubes}

9 Lorentzians were fitted to the experimental spectrum of SWNTs in RBM region (Fig. 1,a), parameters of which are presented in Table 1. RR spectrum of SWNTs produced by HiPCO method was observed by some scientific groups, so every band may be assigned to nanotubes of certain chirality [26,32,33]. Low-frequency bands with peaks at 198.9 and $221.0 \mathrm{~cm}^{-1}$ are attributed to metallic tubes while those in the range of $250-300 \mathrm{~cm}^{-1}$ correspond to semiconducting SWNTs at $\mathrm{He}-\mathrm{Ne}$ laser excitation $(1.96 \mathrm{eV})$ [26]. Table 1 presents the band peak position $(\omega)$, value of which gives nanotubes chirality and diameters. In this Table for each RBM frequency of metallic and semiconducting nanotubes corresponding electronic transitions are also presented, values of which are close to the laser energy $(1.96 \mathrm{eV})$. In the case of metallic nanotubes, $E_{m}^{11}$ denotes the electronic transition between the first peaks of van Hove singularities in the valence and conduction bands, and $E_{S}^{22}$ indicates the electronic transition between the second peaks of van Hove singularities of semiconducting nanotubes. Values of $E_{m}^{11}, E_{S}^{22}$ and relative intensity of lines (shown in brackets) presented in Table 1 were taken from Table in Ref. 32. These energies were obtained for individual HiPCO SWNTs in aqueous suspension with SDS.

Table 1. Peak frequency $\left(\omega, \mathrm{cm}^{-1}\right)$ and area (normalized to $G^{+}$ band) (in brackets) of Lorentzians used to fit the RBM band in Raman spectra of SWNT bundles and SWNT:DNA film at $295 \mathrm{~K}$; electronic transition between first pairs of van Hove singularities of metallic $\left(E_{m}^{11}\right)$ and second ones semiconducting $\left(E_{S}^{22}\right)$ nanotubes $(\mathrm{eV})$ and intensity (in brackets), chirality $(n, m)$ and diameters $(d, \mathrm{~nm})$ of nanotubes determined experimentally from Raman spectrum of semiconducting and metallic HiPCO SWNTs in SDS aqueous suspension [32] taken near with a laser excitation energy $(1.96 \mathrm{eV})$.

\begin{tabular}{c|c|c|c|c}
\hline \hline $\begin{array}{c}\text { SWNT } \\
(295 \mathrm{~K})\end{array}$ & $\begin{array}{c}\text { SWNT:DNA } \\
(295 \mathrm{~K})\end{array}$ & \multirow{2}{*}{$E_{m}^{11}, E_{S}^{22}}$, & & \\
\cline { 1 - 2 }$\omega, \mathrm{cm}^{-1}$ & $\begin{array}{c}\omega, \mathrm{cm}^{-1} \\
\left(S_{\mathrm{RBM}} / S_{G^{+}}\right)\end{array}$ & $\mathrm{eV}[32]$ & & \\
$\left(S_{\mathrm{RBM}} / S_{G^{+}}\right)$ & & $1.93(2.5)$ & $(13,4)$ & 1.206 \\
& & $2.02(0.5)$ & $(9,9)$ & 1.221 \\
$195.2(4.3)$ & $194.5(3.6)$ & $1.94(3.6)$ & $(14,2)$ & 1.183 \\
& & $1.91(0.7)$ & $(15,0)$ & 1.175 \\
$201.6(4.3)$ & $200.9(4.6)$ & $2.07(0.9)$ & $(10,7)$ & 1.159 \\
$216.0(1.5)$ & $215.8(0.4)$ & $2.08(2.6)$ & $(12,3)$ & 1.077 \\
$221.5(6.2)$ & $221.4(6.2)$ & $2.06(1.5)$ & $(13,1)$ & 1.060 \\
$253.8(2)$ & $254.3(6.2)$ & $1.95(3.6)$ & $(10,3)$ & 0.924 \\
$258.8(6.3)$ & $258.9(2.7)$ & $2.03(9.8)$ & $(11,1)$ & 0.903 \\
$266.0(0.4)$ & $266.5(1.0)$ & $1.91(2.6)$ & $(7,6)$ & 0.883 \\
$285.4(1.8)$ & $285.5(2.8)$ & $1.92(18.3)$ & $(7,5)$ & 0.818 \\
$299.7(0.2)$ & $299.8(0.2)$ & $1.86(36)$ & $(8,3)$ & 0.772 \\
\hline \hline
\end{tabular}

As was shown earlier, due to the strong van der Waals tube-tube interaction in bundles, $E_{i i}$ lowers by 70-150 meV [11,12] (depending on nanotube chirality). Thus, for our SWNTs in bundles, energy values are lower by $80-100 \mathrm{meV}$ (on average) than those presented in Table 1. The integral intensity (area, indicated in brackets near the frequency value) of every band (Table 1) was normalized to that of the most intensive tangential band ( $G^{+}$band). As the bands corresponding to metallic nanotubes with the maximum at 198.9 and $221.0 \mathrm{~cm}^{-1}$ are rather broad and have an asymmetric form, therefore, each of them was fitted with two Lorentzians. Thus, the band at $198.9 \mathrm{~cm}^{-1}$ was described with two lines with peaks at 195.2 and $201.6 \mathrm{~cm}^{-1}$, the first of them can be assigned to three nanotubes with different chirality among them, the most intensive line is attributed to (14.2) nanotube. $E_{m}^{11}$ of 
this nanotube is located at $1.94 \mathrm{eV}$ which is lower than the laser energy $(1.96 \mathrm{eV})$. A possible contribution of two other nanotubes with $(13,4)$ and $(9,9)$ chiralities into the intensity of the line at $195.2 \mathrm{~cm}^{-1}$ should be taken into account too. If to compare $E_{m}^{11}$ with the laser energy, the first nanotube has lower but the second one has higher transition energies, respectively (see Table 1). The line with the peak at $201.6 \mathrm{~cm}^{-1}$ can be assigned to $(15,0)$ and $(10,7)$ nanotubes with $E_{m}^{11}$ located lower and higher than $1.96 \mathrm{eV}$, respectively.

Two Lorentzian functions with peaks at 216.0 and $221.5 \mathrm{~cm}^{-1}$ were fitted to the band with the maximum at $221.0 \mathrm{~cm}^{-1}$, that can be attributed to nanotubes with chiralities $(12,3)$ and $(13,1)$, respectively. $E_{m}^{11}$ of these nanotubes is located somewhat higher than the laser energy (Table 1).

The situation with the assignment of bands of semiconducting nanotubes to the certain chirality is simpler than for metallic nanotubes (Table 1), because these bands are narrow and their peaks are separated in the spectrum. It should be noted that among these SWNTs only one band (at $258.8 \mathrm{~cm}^{-1}$ ) is assigned to the tube with $(11,1)$ chirality, the electronic transition of which is located higher than the laser energy. The electronic transition of other nanotubes is lower than $1.96 \mathrm{eV}$.

Energies of electronic transitions of individual nanotubes covered with DNA in aqueous suspension are about 10-80 meV lower than $E_{i i}$ of nanotubes in SDS surrounding [7,34-36]. This is caused by a stronger polymer interaction with the nanotube surface or by an incomplete covering of the nanotube surface with the polymer. As a result, polymer free surface will be in contact with water molecules, and this, in part, lowers the electronic level [37]. In comparison with aqueous suspension of SWNT:DNA, the magnitude of $E_{i i}$ in film decreases further by about 10-20 meV [7]. Evidently, such energy lowering is a result of increasing the interaction energy between the polymer and the nanotube in the solid state as well as of the formation of bundles in which, however, nanotubes are separated with the polymer. One confirmation of this statement that the interaction between the nanotube and DNA in the solid state is stronger is based on the enhancement of the electrostatic interaction in the solid state because: i) the dielectric constant of water is much higher than that of air and ii) the distance between charges in the film is decreased in comparison with solution where water molecules reduces interaction between the nanotube and DNA. It should be noted that this energy lowering is weaker than at the nanotube bundle formation. At the same time, the nanotube interaction with the substrate should decrease $E_{i i}$ too, and this fact must be taken into account. Thus, electronic levels of nanotubes in the film with DNA are energetically higher than those in bundles (by about 50-100 meV). This energy analysis needs to consider the influence of the resonance conditions on the band intensity. It is known that the width of the resonance window is wider for nanotubes in bundles than for isolated SWNTs [11,12].

RR spectrum of SWNT:DNA films in the range of RBM is of common similarity with the spectrum of nanotubes in bundles (Fig. 1,a). We note 25\% enhancement of the semiconducting nanotube intensity in comparison with metallic SWNTs. Thus, the M:S (metallic:semiconducting) ratio of integrated intensities for RBM associated with metallic tubes to those of semiconducting ones in bundles and film with DNA are 1.52 and 1.15, respectively. Such an enhancement can be explained both by changes in resonance conditions and DNA selectivity to interact preferably with nanotubes of the certain diameter [38,39].

For metallic nanotubes in film with DNA, the intensity of the band with a maximum at $198.8 \mathrm{~cm}^{-1}$ frequency increased in comparison with the band intensity at $221.4 \mathrm{~cm}^{-1}$. In semiconducting nanotubes an essential rise of the band intensity at $254.3 \mathrm{~cm}^{-1}$ and the intensity drop of the band at $258.9 \mathrm{~cm}^{-1}$ are observed. Such a redistribution of band intensities can be attributed to changes in resonance conditions for different nanotubes in bundles and film with DNA. Some insight into the manifestation of these conditions in Raman spectra may be got from Fig. 2 which presents the energetic scheme of resonant windows for two semiconducting SWNTs (in various environments) with regard to the laser energy $(1.96 \mathrm{eV})$. The vertical line denotes the laser energy and shapes of the window for individual SWNTs in aqueous solutions with SDS (bold line) or with DNA (dashed line) and in bundles (thin line). To describe this window, a Lorentzian function was used. Two nanotubes with index chirality $(10,3)$ (Fig. 2,a) and $(11,1)$ (Fig. 2,b) were selected for this scheme. These nanotubes have RBM frequencies at 254.3 and $258.9 \mathrm{~cm}^{-1}$ in the SWNT:DNA film, respectively. For the first nanotube $E_{S S}^{22}$ is lower $(1.95 \mathrm{eV})$ and for the second one is higher $(2.03 \mathrm{eV})$ than the laser energy. $E_{s s}^{22}$ for these nanotubes in bundles was taken by $80 \mathrm{meV}$ (on average) lower than obtained for individual SWNTs. Average values of the resonance window width (at half of height) were taken as $80 \mathrm{meV}$ for nanotubes in bundles [12], $40 \mathrm{meV}$ for SWNT:DNA film $[35,36]$ and $30 \mathrm{meV}$ for individual nanotubes in aqueous solutions with SDS [32]. If to proceed from bundles to film with DNA, the integral intensity of the line at $254.3 \mathrm{~cm}^{-1}$ must increase due to better resonance conditions and, on the contrary, the integral intensity of the line at $258.9 \mathrm{~cm}^{-1}$ must decrease because of $E_{S S}^{22}$ of this tube escapes from the resonance. Just the same intensity behavior of selected bands has been observed in experimental spectra.

Detailed analysis provided for two semiconducting nanotubes, with $E_{S S}^{22}$ being lower or higher than the laser energy, can be applied to other nanotubes. Thus, integral intensity of bands at 266.5, 285.4 and $299.8 \mathrm{~cm}^{-1}$ will increase if to proceed from bundles to the film with DNA that is well seen in RR nanotubes spectra (Fig. 1,a). 

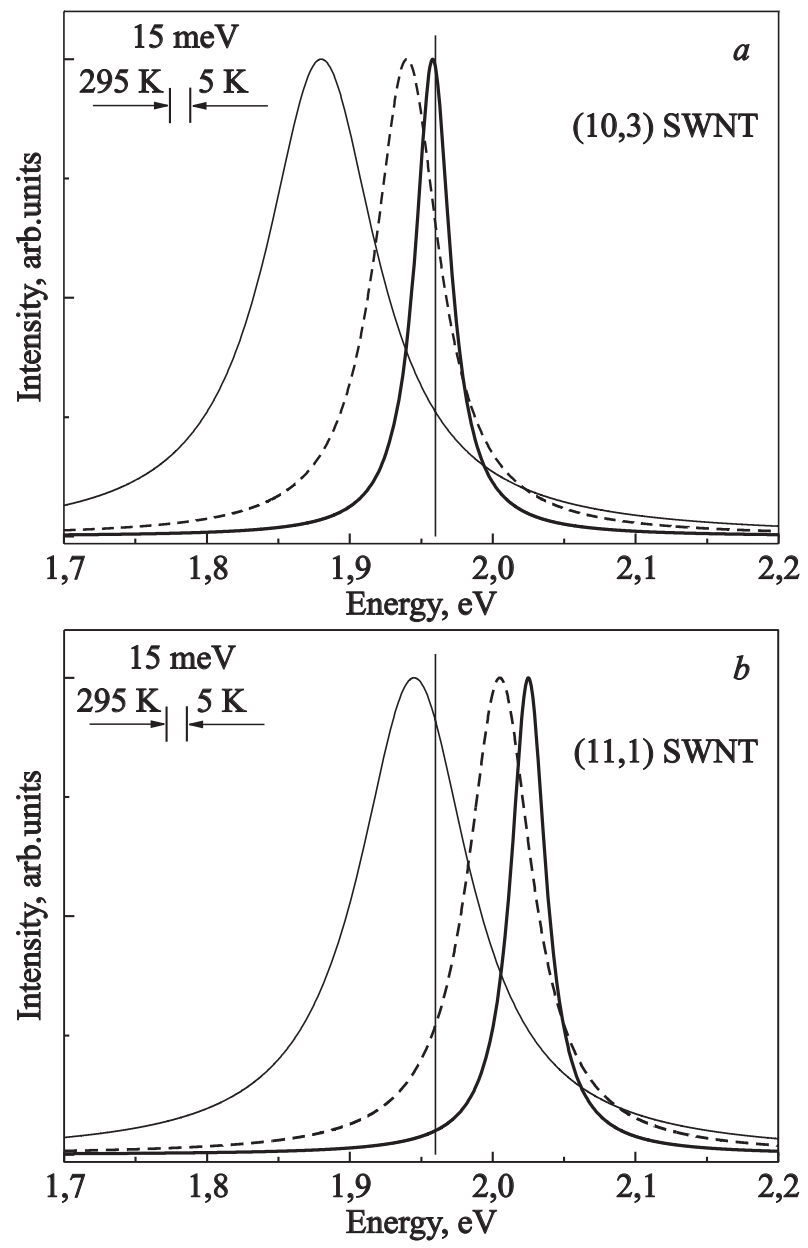

Fig. 2. Scheme of resonant windows of semiconducting nanotubes with different chirality $(10,3)(a)$ and $(11,1)(b)$ : arranged in bundles (thin curve), aqueous suspension of individual nanotubes in SDS (bold curve) and DNA surrounding (dashed curve) located close to energy excitation $(1.96 \mathrm{eV})$ of $\mathrm{He}-\mathrm{Ne}$ laser (straight line). Lorentzian curves were used to describe a contour of resonant windows.

In the case of metallic nanotubes the similar analysis is complicated as two lines are taken to fit to the experimental band and each line can be assigned to 2-3 nanotubes of different chirality, $E_{m}^{11}$ value of which is lower or higher than the laser energy. The ratio of integral intensities of low- and high-frequency bands are 1.12 in RR spectra of metallic nanotubes in bundles and 1.24 in spectra of SWNT:DNA film. Table 1 demonstrates that this ratio change is mainly caused by the intensity decrease of the high-frequency band. As $E_{m}^{11}$ of nanotubes attributed to this band has a higher value than the laser energy, such an intensity decrease (proceeding from bundles to the film with DNA) is expected. The integral intensity of the band at $198.9 \mathrm{~cm}^{-1}$ is somewhat weaker for SWNT:DNA films too in comparison with that of bundles though $(14,2)$ nanotube has a lower value of $E_{m}^{11}$ than the laser energy, and in this case the intensity rise could be expected. However, this has not occurred, possibly, because the contribution of other nanotubes into the integral intensity of this band must be taken into account.

Comparing the band position in RBM spectra of nanotubes in bundles and in the film with DNA reveals that the spectral shift does not exceed $0.7 \mathrm{~cm}^{-1}$. It should be noted that for metallic nanotubes in the film with DNA the downshift is observed, and in the case of semiconducting SWNTs the band is upshifted or the band position is not changed practically. A small shift of peaks of RBM bands of SWNT:DNA film versus bands of nanotube bundles into different sides for semiconducting and metallic nanotubes indicates that DNA interactions with various types of SWNTs are different. In recent article of Prof. S. Iijima with coworkers [40] it was concluded that ssDNA selectively interacts with the metallic nanotubes and modifies the electronic structure.

Is it possible to explain the evolution in the intensity of RBM bands proceeding from nanotubes in the bundle to a SWNT:DNA film, considering only changes in resonance conditions? Let us to compare changes in the intensity of the band at $221.4 \mathrm{~cm}^{-1}$, attributed to two metallic nanotubes with $E_{m}^{11}$ that is higher than $1.96 \mathrm{eV}$, and in the intensity of the semiconducting nanotube at $258.9 \mathrm{~cm}^{-1}$ with the similar value of energy transition $\left(E_{S}^{22}\right)$. The decrease of the last band integral intensity going from bundles to film with DNA is $57 \%$ but the band intensity of metallic nanotubes weakens only by $14 \%$. In part, such a difference in the intensity weakness can be explained with the different resonance window the width of which depends on the tube chirality $[11,12]$. But it seems to us that the main reason of this different intensity weakening is caused by various DNA interactions with metallic and semiconducting nanotubes. As for one type of SWNTs, this interaction depends on the diameter/chirality too $[38,39]$.

\section{Tangential mode of nanotubes}

The tangential mode of nanotubes (Fig. 1,b) consists of two components: a narrow band near $1590 \mathrm{~cm}^{-1}$ and a broadened low-frequency $1520-1570 \mathrm{~cm}^{-1}$ band $\left(G^{+}\right.$and $G^{-}$bands, respectively). These bands are due to $\mathrm{C}-\mathrm{C}$ vibration along the nanotube axis (LO phonon) and with the carbon atom vibration in the tangential direction with regard to the tube axis (TO phonon). The high-frequency component $\left(1560-1620 \mathrm{~cm}^{-1}\right)$ is observed not only in SWNTs spectrum but in the Raman spectra of multiwalled carbon nanotubes and of graphite and graphene as well. The presence of the low-frequency component $G^{-}$ which appears due to curvature and confinement is a feature of SWNTs only. It should be noted that the observed spectrum is a result of the spectrum superposition of both semiconducting and metallic type nanotubes with different diameters. To describe the two components of the tangential mode, the nanotube spectrum was fitted with the minimal number of approximation functions: two curves for each nanotubes type. 
The low-frequency component of the $G^{-}$band is wide with an asymmetric form, which is strongly broadened in the range of lower frequencies. As was shown, the sloping lower-frequency front of the band is conditioned with metallic nanotubes. The shape of such a band is well described by Breit-Wigner-Fano (BWF) function [41]: $I(\omega)=I_{0}\left\{1+\left(\omega-\omega_{0}\right) / q \Gamma\right\}^{2} /\left\{1+\left[\left(\omega-\omega_{0}\right) / \Gamma\right]^{2}\right\}$, where $I_{0}$, $\omega_{0}, \Gamma$ and $q$ are intensity, the BWF peak frequency, broadening parameter, and the asymmetry parameter, respectively. The asymmetric line shape of the $G^{-}$band has been attributed to interference scattering between an electronic continuum present in metallic tubes and the $G^{-}$Raman active band. This BWF band is of a weak intensity in individual nanotubes [42] and is practically absent in SWNT:DNA aqueous suspensions after ultracentrifugation $[7,43]$. The intensity of $G^{-}$band rises significantly in bundles as it was supposed earlier due to the strong influence of the tube-tube interaction on coupling of phonons with plasmons in metallic nanotubes [42].

However, recent detailed theoretical models showed that the $G^{-}$peak in metallic nanotubes is caused by the LO mode, but not the TO mode as in semiconducting SWNTs [19-23]. The LO mode is softened by a Kohn anomaly at the point $\Gamma$ in the phonon dispersion. This model predicates that the $G^{-}$band in metallic SWNTs is due to coupling between phonons and electron-hole pairs, contrary to the earlier theory of plasmon-phonon coupling. The KA occurs in the LO phonon branch because the LO phonon distorts the lattice such so that a dynamic band gap is induced in the electronic band structure. This accompanies with the energy decrease of the electrons near the Fermi point, and the energy required to distort the lattice is reduced too, leading to phonon softening. Thus, LO phonon in RR spectrum of metallic nanotubes has a lower frequency than TO phonon. In the spectrum of semiconducting nanotubes the assignment of bands is opposite: the frequency for LOphonons is higher than for TO ones. This model was supported by the experimental study [15-18].

For two investigated samples the low-frequency component of $G$ mode was approximated with one BWF and one Lorentzian functions. Unlike aqueous suspension of SWNT:DNA, in its film the band intensity described with BWF function increases $[7,44]$ but its intensity is lower than that of nanotubes in bundles. The second band described with Lorentzian function was assigned to semiconducting nanotubes with the maximum at $1557.0 \mathrm{~cm}^{-1}$, in SWNT:DNA film this band is by $2.6 \mathrm{~cm}^{-1}$ is upshifted versus this band in SWNT bundles. The similar evolution with the $\mathrm{G}^{+}$band was observed too: its peak is upshifted by $4.3 \mathrm{~cm}^{-1}$. Two Lorentzians were fitted to this band, the low-frequency line was attributed to nanotubes with metallic conductivity and the line of higher frequency is related to semiconducting ones [45]. The value of the band peak position and its area are indicated (in brackets) near the bands in Fig. 1, $b$. The area of each line was normalized to the total area of all bands of $G$ mode, being taken as $100 \%$. It should be noted that the width of $G^{+}$band increased in SWNT:DNA films. Most likely, the possible reason of such an increasing is the nanotube interaction with the polymer which structure includes the charged group, or other reason can be caused by the inhomogeneous broadening because of the disordering arrangement of nanotubes in the film. The important parameter characterizing the bundle formation is the ratio of areas of high- and low-frequency components of $G$ mode, upon the bundle splitting, this ratio increases [42]. In SWNT:DNA film the integral intensity of the band, described with BWF function, lowers by about $30 \%$.

One possible reason of $G^{+}$band upshift in SWNT:DNA film may be caused by the transfer of the charge between the nanotube and adsorbed molecule. Upon the charge transfer from the nanotube to the molecule, the $G^{+}$band is upshifted, and, when the electron transfer occurs in the opposite direction, this band is downshifted [41,46]. A single-stranded DNA chain forms a helically wrapped hybrid structure around the nanotube, in which the nitrogen bases are extended from the backbone and stacked onto the nanotube surface $[3,4]$. The interaction between bases and the nanotube surface is accompanied by the weak charge transfer. However, as recent $a b$ initio calculations were demonstrated, a weak charge transfer takes place from bases to the nanotube and the $G^{+}$mode softening is observed in RR spectrum of nanotubes covered with bases [47].

The other possible reason of $G^{+}$band upshift in SWNT:DNA film may be connected with the polymer pressure onto the nanotube. Such an assumption is reasonable as the polymer wraps around the nanotube and this adsorption is very strong both in water and in film but the interaction between the nanotube and DNA in the solid state is stronger. According to results of investigations on the external pressure effect on RR spectra of carbon nanotubes $[48,49]$, the $G^{+}$band is shifted to the high-frequency region upon pressure onto the nanotube. In any case, the upshift of $G$ mode frequency indicates that $\mathrm{C}-\mathrm{C}$ bond force constant of nanotubes in film with DNA becomes stronger relatively to this constant in bundles.

\section{Effect of temperature lowering on Raman spectra of carbon nanotubes in bundles and in film with DNA}

Figure 3 presents spectral evolution observed in nanotube bundles upon the temperature lowering from 295 to $5 \mathrm{~K}$. As with RR spectra of these nanotubes at room temperature, the low-temperature spectrum was fitted by the same number of Lorentzian and BWF functions too.

In RR spectrum of nanotubes in bundles at $T=5 \mathrm{~K}$ the intensities of RBM bands corresponding to metallic nanotubes decrease relatively to those of semiconducting nanotubes, for example, M:S ratio is equal to 0.6 but at $295 \mathrm{~K}$ it is 1.52 . At the same time the intensity of the metallic nano- 

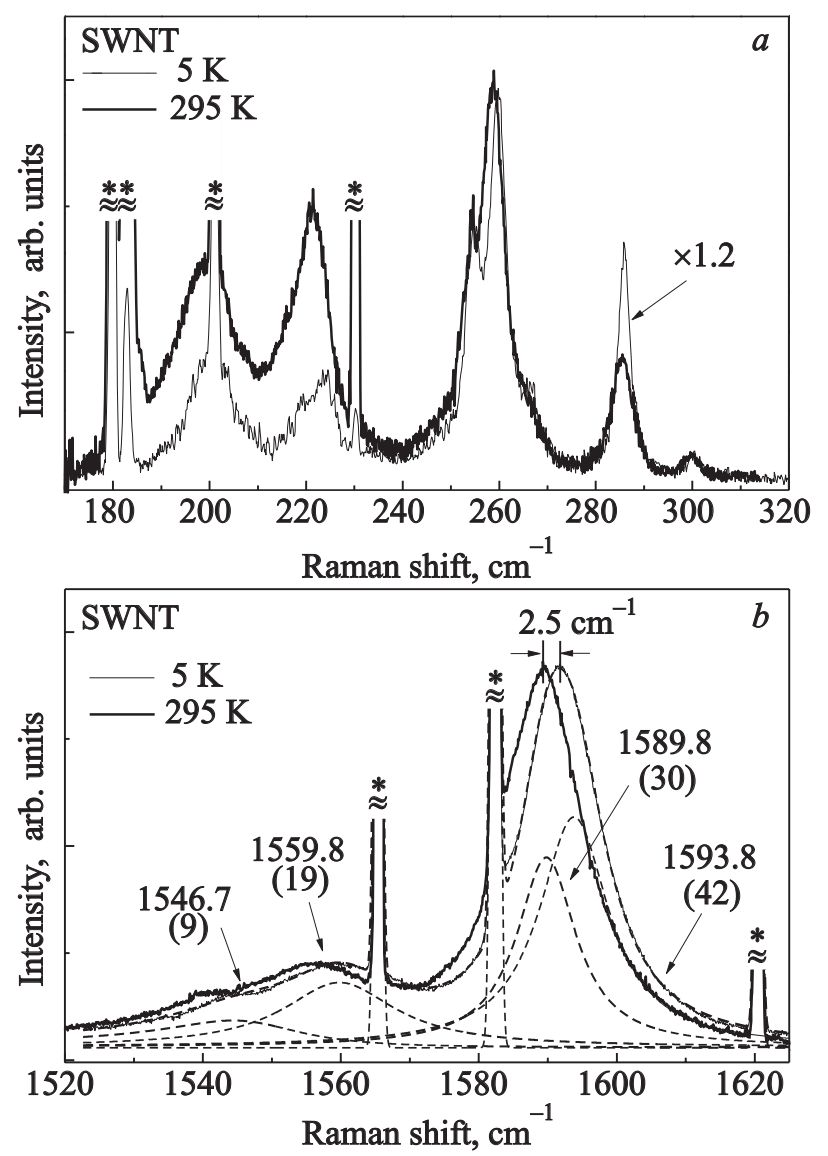

Fig. 3. Raman spectrum in range of RBM ( $a$ ) and $G$ mode $(b)$ of SWNT bundles at $5 \mathrm{~K}$ (bold curve). Curves described by Lorentzian and BWF (a low-frequency band of $G$ mode) functions were used for fitting to the experimental spectrum (dashed lines). Value of band peak position and its area (in brackets) is indicated in this Figure close to the location of bands. For comparison the Raman spectrum of this sample obtained at $295 \mathrm{~K}$ (bold curve) was shown.

tube band with the maximum at $222.5 \mathrm{~cm}^{-1}$ lowers more significantly in comparison with the band at $200.7 \mathrm{~cm}^{-1}$, and, as a result, the ratio of integral intensities of low- and high-frequency bands increased from 1.12 at $295 \mathrm{~K}$ to 1.55 (Table 2).

As with the temperature lowering the population of phonon states in nanotubes decreases, this results in the increase in the energy of electronic transitions (see, for example, [14,50]). The value of $E_{i i}$ increase with temperature about $10-20 \mathrm{meV}$ (this value is different for metallic and semiconducting nanotubes). This energetic evolution changes resonance conditions for nanotubes. So, SWNT with $E_{i i}$ more than the laser excitation energy will leave the resonance window, and the intensity of RBM bands of such nanotubes will weaken, and, to the contrary, the intensity of RBM bands of nanotubes with $E_{i i}$ lower than $1.96 \mathrm{eV}$ will strengthen.

The temperature drop results in an upshift of RBM bands. The shift value for metallic nanotubes is $1.1-3.5 \mathrm{~cm}^{-1}$, for semiconducting nanotubes this upshift is lower (about $1 \mathrm{~cm}^{-1}$ ). It should be noted that similar spectral upshift upon the temperature decrease was observed for isolated nanotubes too [28]. At low temperature the RBM bands became narrow, for some nanotubes this width value (at half of height) reaches $3 \mathrm{~cm}^{-1}$.

Table 2. Peak frequency $\left(\omega, \mathrm{cm}^{-1}\right)$ and area (normalized to $G^{+}$ band) (in brackets) of Lorentzian lines used to fit the RBM band in Raman spectra of SWNT bundles and SWNT:DNA film at $5 \mathrm{~K}$; difference $\left(\Delta(295-5 \mathrm{~K}), \mathrm{cm}^{-1}\right)$ between peaks of Lorentzians determined from the fitting to RBM band in Raman spectra obtained at 5 and $295 \mathrm{~K}$.

\begin{tabular}{c|c|c|c}
\hline \hline \multicolumn{2}{c|}{ SWNT $(5 \mathrm{~K})$} & \multicolumn{2}{c}{ SWNT:DNA(5 K) } \\
\hline $\begin{array}{c}\omega, \mathrm{cm}^{-1} \\
\left(S_{\mathrm{RBM}} / S_{G^{+}}\right)\end{array}$ & $\begin{array}{c}\Delta(295-5 \mathrm{~K}), \\
\mathrm{cm}^{-1}\end{array}$ & $\begin{array}{c}\omega, \mathrm{cm}^{-1} \\
\left(S_{\mathrm{RBM}} / S_{G+}\right)\end{array}$ & $\begin{array}{c}\Delta(295-5 \mathrm{~K}), \\
\mathrm{cm}^{-1}\end{array}$ \\
\hline \hline $197.8(1.4)$ & -2.6 & $197.6(0.5)$ & -3.1 \\
$202.7(2)$ & -1.1 & $202.6(0.7)$ & -1.7 \\
$219.5(1.1)$ & -3.5 & $217.6(0.1)$ & -1.8 \\
$224.2(1.1)$ & -2.7 & $223.8(0.5)$ & -2.4 \\
$254.6(2.3)$ & -0.8 & $255.1(3.0)$ & -0.8 \\
$259.8(4.3)$ & -1.0 & $259.9(0.5)$ & -1.0 \\
$266.7(0.6)$ & -0.7 & $266.9(0.8)$ & -0.4 \\
$285.9(2)$ & -0.5 & $286.1(2.1)$ & -0.6 \\
$300.1(0.2)$ & -0.4 & $300.8(0.1)$ & -1.0 \\
\hline \hline
\end{tabular}

As well, the decrease in $T$ effects on the RR spectrum of nanotubes in bundles in the region of the tangential mode. $G^{+}$band fitted by two Lorentzian functions which demonstrate 2.5 and $2 \mathrm{~cm}^{-1}$ upshifts, for the low- and highfrequency bands, respectively. When $T$ is lowered, an average upshift is $2.5 \mathrm{~cm}^{-1}$, this gives an estimated average temperature coefficient $d \omega / d T \simeq-0.0083 \mathrm{~cm}^{-1} / \mathrm{K}$ for the $G^{+}$peak of semiconducting tubes in bundles. This value is close to the temperature coefficient in isolated nanotubes, being determined as $-0.011 \mathrm{~cm}^{-1} / \mathrm{K}$ [28]. It should be noted that, for graphene, this coefficient is twice higher [28]. The upshift of the band fitted by BWF does not exceed $3.6 \mathrm{~cm}^{-1}$. The integral intensity of the last band decreases at low temperature (Fig. 3).

The temperature effect on Raman frequencies of a material is conditioned with the anharmonic terms in the bond potential energy of the nanotube lattice, induced by its thermal expansion upon the temperature rise. Thus, the temperature increase results in softening of the force constant of $\mathrm{C}-\mathrm{C}$ bond, and the phonon frequency becomes lower [51]. Such a behavior of peaks of RBM and $G$ bands of SWNTs is observed, but the spectral shift of these bands is different, depending on the type of the nanotube conductivity.

To consider the effect of the temperature lowering on RR spectrum of metallic nanotubes, besides an inharmonic term, the contribution of the phonon and electron coupling must be taken into account. The population of the electro- 

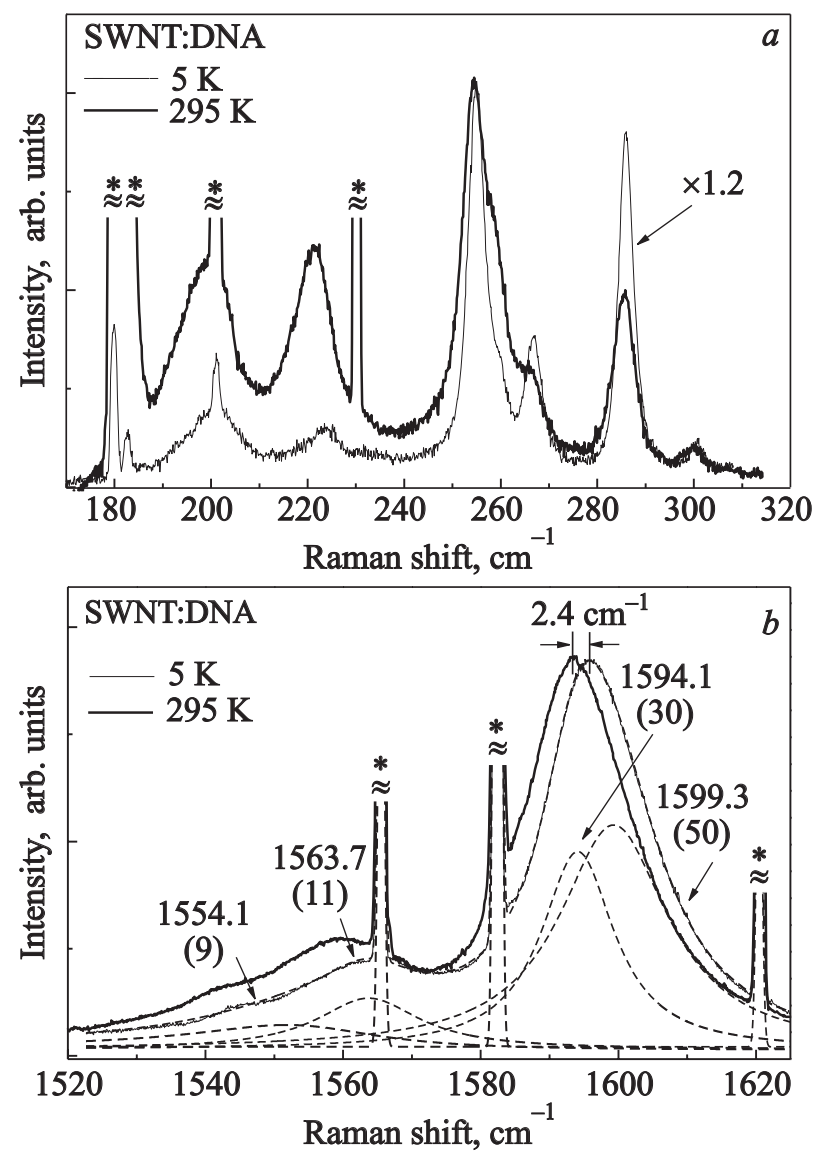

Fig. 4. Raman spectra in range of RBM $(a)$ and $G$ mode $(b)$ of SWNT:DNA film at $5 \mathrm{~K}$ (thin curve). Curves described by Lorentzian and BWF (a low-frequency band of $G$ mode) functions were used to fit the experimental spectra (dashed lines) obtained at this temperature. Numbers indicated in this Figure close to the bands denote the value of band peak position and its area (in brackets). For comparison the Raman spectra of this sample obtained at $295 \mathrm{~K}$ were shown.

nic states with the temperature increase is determined by Fermi-Dirac distribution which influences the shape of Kohn anomaly. With the temperature rise the significance of this anomaly weakens. Thus, the effect of temperature becomes apparent in modification of the electronic screening, as a consequence, LO phonon frequency is changed that can be observed in RR spectrum [15-18]. The effect of LO phonon softening with the temperature rising becomes weaker resulting in the $G^{-}$band frequency increase. However, as the theoretical model predicts [22], this occurs above the $450-500 \mathrm{~K}$ but from helium till room temperatures the $G^{-}$band frequency will decrease. Thus, in our temperature range the frequency downshift induced by electrons will intensify the shift caused by the unharmonic term. As a result, the downshift value of BWF band with temperature is larger than for other bands.

The influence of electron-phonon coupling on RBM of carbon nanotubes was studied recently [17], and the $2 \mathrm{~cm}^{-1}$ softening of the radial breathing mode of metallic nano- tubes due to this coupling was observed, meanwhile, the RBM peak for a semiconducting SWNT shows no appreciable change. By analogy with $G$ mode, we suppose that a larger upshift of RBM of metallic nanotubes versus semiconducting ones, observed at the temperature lowering, is caused by larger electron-phonon coupling for first type of nanotubes. Nevertheless, to understand details of the effect of electron-phonon coupling on RBM, additional experimental and theoretical investigations are necessary.

As at low temperature the intensity of the low frequency component of $G^{-}$band becomes weaker (Fig. 4), therefore, to fit this band with BWF function correctly, we were guided by some rules, namely, at temperature lowering the band width and parameter $1 / q$ increase (does not decrease, at least). These rules were based on detailed temperature analysis of this band in metallic nanotubes, which was fulfilled in the reference [44]. As well, for $G^{-}$bands of SWNT:DNA films the temperature decrease from 295 to $5 \mathrm{~K}$ results in the $7.9 \mathrm{~cm}^{-1}$ upshift for the band fitted with BWF function and for another band this shift is equal to $4.1 \mathrm{~cm}^{-1}$. Fitting to the $G^{+}$band gives the upshift by 1.9 and $2.3 \mathrm{~cm}^{-1}$ for low- and high-frequency Lorentzian curves, respectively. At $5 \mathrm{~K}$ the area of the BWF band decreases relatively to room temperature. It should be noted that the high-frequency spectral shift of $G$ bands observed at $295 \mathrm{~K}$ for SWNT:DNA film versus nanotube bundles is retained at helium temperature too (Fig. 5). $-0.01 \mathrm{~cm}^{-1} / \mathrm{K}$ (5-295 K) value of the temperature coefficient of the SWNT:DNA film is obtained after the determination of the $G^{+}$band shift. The absolute value of the temperature coefficient is slightly larger than the magnitude obtained for nanotubes in bundles.

We try to determine the magnitude of the spectral splitting between two components of the tangential mode for metallic and semiconducting nanotubes $\Delta\left(G^{+}-G^{-}\right)$and to compare their temperature dependences. There are two complications upon these estimations: the first one is caused by the decrease in accuracy of determining the peak position of the $G^{-}$band at low temperature because of weakening the BWF band intensity, the another results from the fact that in our samples the peaks of $G^{+}$and $G^{-}$ bands are attributed to an averaged nanotube, and this reduces slightly the correctness of these estimations. With temperature rising from $5 \mathrm{~K}$ till $295 \mathrm{~K}$ the magnitude of $\Delta\left(G^{+}-G^{-}\right)$for metallic SWNTs increases by 1.9 and $6 \mathrm{~cm}^{-1}$ for nanotubes in bundles and in film with DNA, respectively. Meanwhile, the magnitude of $\Delta\left(G^{+}-G^{-}\right)$for semiconducting SWNTs increases by $1.4 \mathrm{~cm}^{-1}$ with the temperature growth only for SWNT:DNA film, and other sample shows no appreciable change in this splitting. These estimations confirm the significant electron-phonon coupling in metallic nanotubes and coincide with temperature measurements provided by other authors [15-17]. However, additional experiments with more intensive 

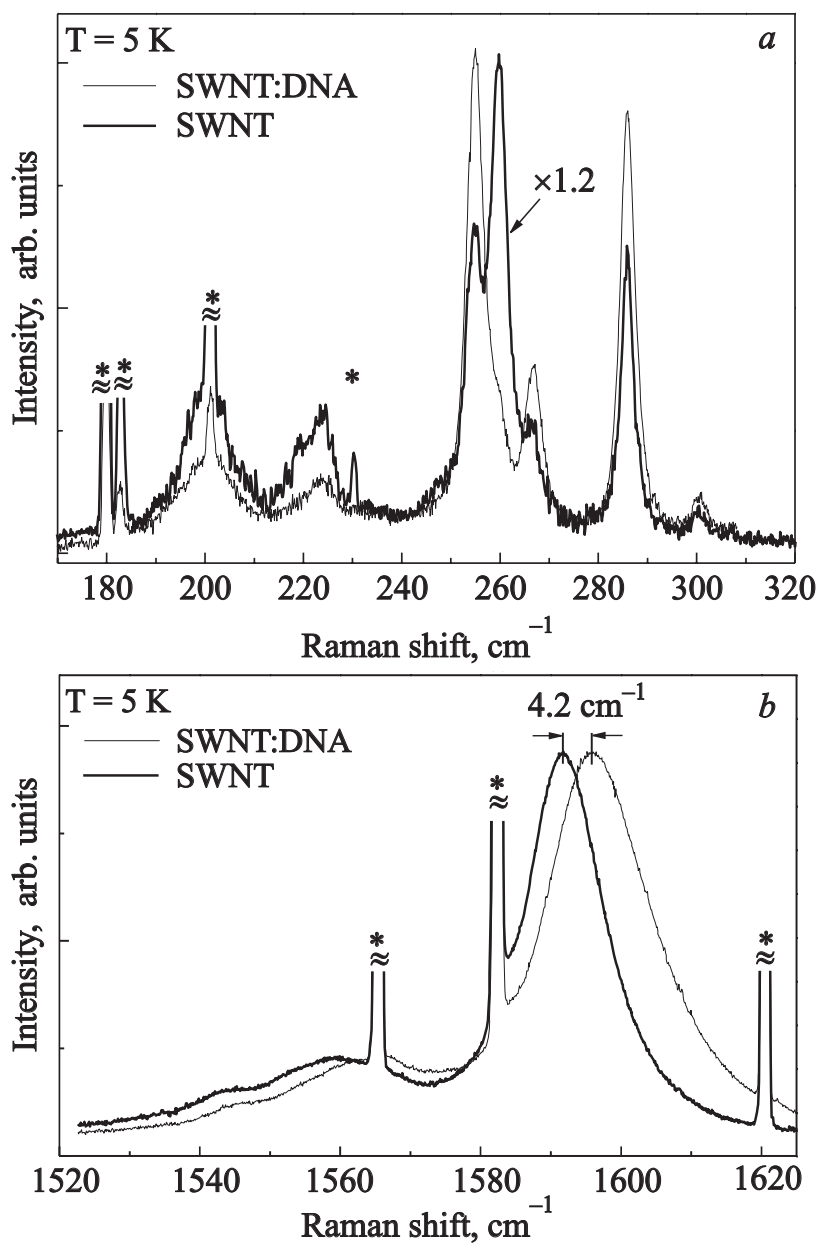

Fig. 5. Raman spectra of SWNT bundles (bold curve) and SWNT:DNA film (thin curve) at $5 \mathrm{~K}:(a)$ range of RBM and $(b)$ $G$ mode.

BWF band in RR spectra and with individual nanotubes should be carried out.

Figure 5 presents RR spectra of SWNT:DNA film and nanotube bundles at $5 \mathrm{~K}$, which permits to compare the spectra evolution of two samples helium and room temperatures (Fig. 1). It is seen that for RBM region the temperature lowering is accompanied by noticeable changes in the intensity redistribution among bands of two samples relatively to $295 \mathrm{~K}$ and can be mainly explained by transformation of resonance conditions. $G$ mode region is characterized with an decrease of BWF band intensity, however, the value of the spectral shift between $G^{+}$band peaks of two samples retains the same as it was observed at $295 \mathrm{~K}$ (Fig. 1). In general, a substitution of the strong interaction between tubes in bundles with a rather strong of the SWNT interaction with the polymer does not result in crucial changes of the nanotube RR spectrum.

\section{Conclusions}

The carbon nanotube-DNA interaction results in the following changes in Raman spectrum of this sample relatively to the spectrum of nanotubes in bundles: increasing of the integral intensity of RBM bands, redistribution of intensities between the bands, which can be explained partly by transformation of resonance conditions, $G$ band is upshifted, and the intensity of the asymmetric band of the low-frequency component of this band weakens in film.

The temperature lowering from 295 to $5 \mathrm{~K}$ is accompanied by the upshift of RBM bands, the magnitude of which is higher for metallic nanotubes than that for semiconducting ones. A larger upshift of RBM of metallic nanotubes is most probably caused by stronger electron-phonon coupling in these nanotubes. With the temperature decrease the band width becomes narrower, and some band width reaches $3 \mathrm{~cm}^{-1}$ at $5 \mathrm{~K}$. The similar spectral evolution is observed for nanotubes in bundles. Noticeable changes observed in the intensity redistribution among RBM bands of two samples at the decrease in $T$ can be mainly explained by transformation of resonance conditions.

Upon the temperature lowering from 295 to $5 \mathrm{~K}$, the upshift of bands attributed to $G$ mode is observed for two samples but its magnitude is different for metallic and semiconducting nanotubes. Provided estimations confirm the significant coupling between electron and this high-frequency phonon in metallic nanotubes. The temperature coefficient $d \omega / d T$ determined by the temperature shift of $G^{+}$band is equal to $-0.01 \mathrm{~cm}^{-1} / \mathrm{K}$ for NT:DNA film and $-0.0083 \mathrm{~cm}^{-1} / \mathrm{K}$ for nanotubes in bundles. It should be noted that the spectral shift value between of $G^{+}$band peaks of two samples at $5 \mathrm{~K}$ retains the same as it was observed at $295 \mathrm{~K}$

In general, it can be concluded that temperature changes observed in RR spectra of SWNT:DNA film and nanotubes in bundles are similar. It results from the fact that the strong interaction between tubes in bundles is replaced with a rather strong SWNT interaction with the polymer.

\section{Acknowledgment}

We are grateful to Dr. U. Dettlaff-Weglikowska (MaxPlanck-Institute for Solid State Research) for purified SWNTs, to PhD. A. Peschanskii (ILTPE) for helpful discussions and to V. Leontiev for samples preparation. This work was partially supported by Grants 4950 of the Science and Technology Center in Ukraine and National Academy of Sciences of Ukraine.

1. Nanomaterials for Biosensors, C. Kumar (ed.) Wiley-VCH Verlag GmbH\&Co./KGaA (2007).

2. Applied Physics of Carbon Nanotubes, S. Rotkin and S. Subramoney (eds.) Springer Berlin Heidelberg New York (2005).

3. M.J. O'Connell, P. Boul, L.M. Ericson, Ch. Huffman, Y. Wang, E. Haros, C. Kuper, J. Tour, K.D. Ausman, and R.E. Smalley, Chem. Phys. Lett. 342, 265 (2001).

4. M. Zheng, A. Jagota, E. Semke, B. Diner, R. Mclean, S. Lustig, R. Richardson, and N. Tassi, Nature Mater. 2, 338 (2003).

5. H. Cathcart, S. Quinn, V. Nicolosi, J.M. Kelly, W.J. Blau, and J.N. Coleman, J. Phys. Chem. C111, 66 (2007).

Fizika Nizkikh Temperatur, 2010, v. 36, No. 5 
6. V.A. Karachevtsev, G.O. Gladchenko, M.V. Karachevtsev, V.A. Valeev, V.S. Leontiev, and O.S. Lytvyn, Chem. Phys. Phys. Chem. 9, 2872 (2008).

7. V.A. Karachevtsev, A.Yu. Glamazda, U. Dettlaff-Weglikowska, V.S. Leontiev, P.V. Mateichenko, S. Roth, and A.M. Rao, Carbon 44, 1292 (2006).

8. H. Kawamoto, T. Uchida, K. Kojima, and M. Tachibana, J. Appl. Phys. 99, 094309 (2006).

9. A.M. Rao, E. Richter, S. Bandow, B. Chase, P.C. Eklund, K.A. Williams, S. Fang, K.R. Subbaswamy, M. Menon, A. Thess, R.E. Smalley, G. Dresselhaus, and M.S. Dresselhaus, Science 275, 187 (1997).

10. M.S. Dresselhaus, G. Dresselhaus, R. Saito, and A. Jorio, Phys. Rep. 409, 47 (2005)

11. J.S. Park, Y. Oyama, R. Saito, W. Izumida, J. Jiang, K. Sato, C. Fantini, A. Jorio, G. Dresselhaus, and M.S. Dresselhaus, Phys. Rev. B74, 165414 (2006).

12. S.K. Doorn, J. Nanosci. Nanotech. 5, 1023 (2005).

13. C. Thomsen and S. Reich, Raman Scattering in Carbon Nanotubes in Light Scattering in Solid IX, M. Cardona and R. Merlin (eds.), Springer-Verlag: Berlin Heidelberg (2007).

14. R. Capaz, C. Spataru, P. Tangney, M. Cohen, and S. Louie, Phys. Rev. Lett. 94, 036801 (2005).

15. H. Farhat, H. Son, G.G. Samsonidze, S. Reich, M.S. Dresselhaus, and J. Kong, Phys. Rev. Lett. 99, 145506 (2007).

16. V. Scardaci, S. Piscanec, M. Lazzeri, R. Krupke, F. Mauri, and A.C. Ferrari, cond.-mat.0711.4533v1 (2007).

17. Farhat, K. Sasaki, M. Kalbac, M. Hofmann, R. Saito, M.S. Dresselhaus, and J. Kong, Phys. Rev. Lett. 102, 126804 (2009)

18. M. Fouquet, H. Telg, J. Maultzsch, Y. Wu, B. Chandra, J. Hone, T.F. Heinz, and C. Thomsen, Phys. Rev. Lett. 102, 075501 (2009)

19. M. Lazzeri and F. Mauri, Phys. Rev. Lett. 97, 266407 (2006).

20. V.N. Popov and P. Lambin, Phys. Rev. B73, 085407 (2006).

21. A.C. Ferrari, Solid State Commun. 143, 47 (2007).

22. S. Piscanec, M. Lazzeri, J. Robertson, A.C. Ferrari, and F. Mauri, Phys. Rev. B75, 035427 (2007).

23. N. Caudal, A.M. Saitta, M. Lazzeri, and F. Mauri, Phys. Rev. B75, 115423 (2007).

24. C. Thomsen, S. Reich, A.R. Goni, H. Jantoljak, P.M. Rafailov, I. Loa, K. Syassen, C. Journet, and P. Bernier, Phys. Status Solidi B215, 435 (1999).

25. M.N. Iliev, A.P. Litvinchuk, S. Arepalli, P. Nikolaev, and C.D. Scott, Chem. Phys. Lett. 316, 217 (2000).

26. V.A. Karachevtsev, A.Y. Glamazda, U. Dettlaff-Weglikowska, V.S. Kurnosov, E.D. Obraztsova, A.V. Peschanskii, V.V. Eremenko, and S. Roth, Carbon 41, 1567 (2003).

27. T. Uchida, M. Tachibana, S. Kurita, and K. Kojima, Chem. Phys. Lett. 400, 341 (2004).

28. L. Zhang, Z. Jia, L. Huang, S. O'Brien, and Z. Yu, J. Phys. Chem. C112, 13893 (2008).

29. Y. Zhang, L. Xie, J. Zhang, Z. Wu, and Z. Liu, J. Phys. Chem. C111, 14031 (2007).

30. J. Rintala, O. Herranen, A. Johansson, M. Ahlskog, and M. Pettersson, J. Phys. Chem. C 113, 15398 (2009).
31. U. Dettlaff-Weglikowska, J.-M. Benoit, P.-W. Chiu, R. Graupner, S. Lebedkin, and S. Roth, Curr. Appl. Phys. 2, 497 (2002).

32. J. Maultzsch, H. Telg, S. Reich, and C. Thomsen, Phys. Rev. B72, 205438 (2005).

33. C. Fantini, A. Jorio, M. Souza, M.S. Strano, M.S. Dresselhaus, and M.A. Pimenta, Phys. Rev. Lett. 93, 147406 (2004).

34. M.S. Strano, J. Am. Chem. Soc. 125, 16148 (2003).

35. S.G. Chou, H.B. Ribeiro, E.B. Barros, A.P. Santos, D. Nezich, Ge.G. Samsonidze, C. Fantini, M.A. Pimenta, A. Jorio, F. Plentz Filho, M.S. Dresselhaus, G. Dresselhaus, R. Saito, M. Zheng, G.B. Onoa, E.D. Semke, A.K. Swan, M.S. Ünlü, and B.B. Goldberg, Chem. Phys. Lett. 397, 296 (2004).

36. C. Fantini, A. Jorio, A.P. Santos, V.S.T. Peressinotto, and M.A. Pimenta, Chem. Phys. Lett. 439, 138 (2007).

37. M.S. Strano, M. Zheng, A. Jagota, G.B. Onoa, D.A. Heller, P.W. Barone, and M.L. Usrey, Nano Lett. 4, 543 (2004).

38. S.N. Kim, Z. Kuang, J.G. Grote, B.L. Farmer, and R.R. Naik, Nano Lett. 8, 4415 (2008).

39. Q.-H. Yang, N. Gale, C.J. Oton, F. Li, A. Vaughan, R. Saito, I.S. Nandhakumar, Z.-Y. Tang, H.-M. Cheng, T. Brown, and W.H. Loh, Nanotechnology 18, 405706 (2007).

40. M. Shoda, S. Bandow, Y. Maruyama, and S. Iijima. J. Phys. Chem. C113, 6033 (2009)

41. A.M. Rao, P.C. Eklund, S. Bandow, A. Thess, and R.E. Smalley, Nature 388, 257 (1997).

42. C. Jiang, K. Kempa, J. Zhao, U. Kolb, U. Schlech, T. Basche, M. Burghard, and A. Mews, Phys. Rev. B66, 161404 (2002).

43. H. Kawamoto, T. Uchida, K. Kojima, and M. Tachibana, J. Appl. Phys. 99, 094309 (2006).

44. T. Uchida, M. Tachibana, S. Kutita, and K. Kojima, Chem. Phys. Lett. 400, 341 (2004)

45. A. Jorio, C. Fantini, M. Dantao, M.A. Pimenta, A.G. Souza Filho, Ge.G. Samsonidze, V.W. Brar, G. Dresselhaus, M.S. Dresselhaus, A.K. Swan, M. Unlu, B.B. Colberg, and R. Saito, Phys. Rev B66, 115411 (2002).

46. K.E. Wise, C. Park, E.J. Siochi, and J.S. Harrison, Chem. Phys. Lett. 391, 207 (2004).

47. S.G. Stepanian, M.V. Karachevtsev, A.Yu. Glamazda, V.A. Karachevtsev, and L.J. Adamowicz, J. Phys. Chem. A113, 3621 (2009).

48. U.D. Venkateswaran, E.A. Brandsen, U. Schlecht, A.M. Rao, E. Richter, I. Loa, K. Syassen, and P.C. Eklund, Phys. Status Solidi B223, 225 (2001).

49. S. Lebedkin, K. Arnold, O. Kiowski, F. Hennrich, and M.M. Kappes, Phys. Rev. B73, 094109 (2006).

50. F. Simon, R. Pfeiffer, and H. Kuzmany, Phys. Rev. B74, 121411 R (2006).

51. N.R. Raravikar, P. Keblinski, A.M. Rao, M.S. Dresselhaus, L.S. Schadler, and P.M. Ajayan, Phys. Rev. B66, 235424 (2002). 